\title{
Incorporating Patent Analysis in Axiomatic Design for New Product Development
}

\author{
Wenguang Lin ${ }^{1}$, Renbin Xiao ${ }^{2 *}$, Rongshen Lai ${ }^{1}$, Xiaozhen Guo ${ }^{3}$ \\ ${ }^{1}$ School of Mechanical \& Vehicle Engineering, Xiamen University of Technology, Xiamen 361005, China \\ ${ }^{2}$ School of Artificial Intelligence and Automation, Huazhong University of Science and Technology, Wuhan 430074, China \\ 3 School of Economic \& Management, Xiamen University of Technology, Xiamen 361005, China
}

\begin{abstract}
Axiomatic design theory is widely used in new product development by providing design solutions through mapping between functional requirements and design parameters. However, the theory does not provide a method to help designer obtain and select design parameters. To this end, this paper introduces patent analysis to overcome the deficiency. Firstly, functional requirements are transformed into patent search terms, and design parameters are obtained from patents. Secondly, morphological matrix is used to represent the relationships between target function and multiple design parameters. Thirdly, design parameters with higher patent frequency are chose and combined into a new scheme. Finally, the scheme is evaluated by the independent axiom of Axiomatic Design theory. The methodology is demonstrated and validated with a case study of spa shower.
\end{abstract}

\section{Introduction}

New product development (NPD) are complex processes to bring a new product to market. There are three reasons: Firstly, customer's requirements in the early stage are not clear for unclear statement; Secondly, design parameters are difficult to obtain, especially when designer is lacking experience; Thirdly, Product must avoid violating the granted patents belong to other patentees. These reasons will also lead to low R\&D efficiency and increased market risk. Therefore, it is very important for designers to have a useful design tool.

Axiomatic design (AD) (Suh, 2001) theory is a system design methodology for identifying the relationship between function requirements and design parameters. AD first using Zigzag process to mapping customer requirements(CRs), function requirements (FRs) and design parameters(DPs) from top layer to down layer until some enforceable design solutions are achieved, then using the two axioms to evaluate the solutions, as shown in Fig 1. The theory can help designers to complete product design and help them evaluate product design plans according to performance, robustness, reliability and functionality $(\mathrm{Li}$ and Song et al., 2019) and is welcomed by product developers for its convenient and good visualization(Wang and Zhang et al., 2017). However, the theory is usually powerful tools for experienced designers and hard for unexperienced users for it does not provide a method that how to obtain design parameters. Therefore, it's necessary to introduce other methods to make up to the shortcomings of the theory.

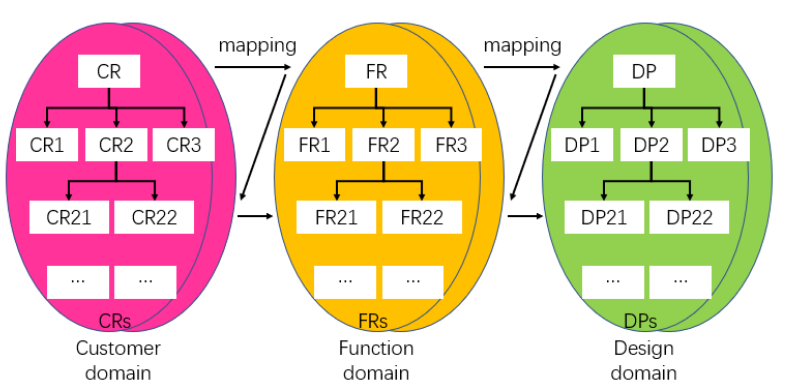

Fig. 1. Zigzag process of $\mathrm{AD}$

In the existing axiomatic design process, design parameters are often obtained from two sources. The first source is designer's skill and knowledge(Kumar and Tandon, 2019). Although designer's skill and knowledge are more reliable than other sources, designer's experience is scare and usually affected by emotion of expert. So is necessary to expand the sources. Although designer's experience is important and reliable for product development, experienced designer is hard to employed and some persons' knowledge are not enough to meet the requirements of diversified product design. The second source is enterprise product information database(He and Zhu et al., 2017). Although large enterprise databases can provide a lot of information for the design process, small and medium-sized enterprises, especially new enterprises, do not have such capabilities. In the early stage of product design, designers need to obtain as much information as possible so as to reduce the risk. Therefore, it is not enough to rely solely on the existing data of individuals or enterprises.

\footnotetext{
* Corresponding author: rbxiao@hust.edu.cn
} 
In recent years, big data mining has been increasingly welcomed by academia and industry. It has been successfully applied in the telecommunications, financial analysis, medical treatment and other fields. In product design, big data mining technology has also been applied. Such as web-based for FRs representation(Yan and Chen et al., 2009), e-commerce product review for FRs analysis(Ireland and Liu, 2018), and cloud-based for design knowledge storage and exchange(Schaefer, 2014).

However, the data involved in the above studies is difficult to obtain, actually. Whether it is network data, commentary data or cloud data, they need to be licensed beforehand, or they will face legal risks. At the same time, the accuracy and objectivity of data may not fully meet the requirements of product development. By contrast, patents are law tools used to protect innovation and ideas, so patents are very important to enterprises(Chang and Trappey et al., 2014). Meanwhile, patents are disclosure documents in the web of national intellectual property bureaus, so they are easy to be legally obtained and more accurate and objective than other data.

Therefore, this paper proposes to use patents data to solve the shortcomings of axiomatic design theory. Through patent analysis, customer's requirements can be abstracted into function requirements, and functional requirements can be quickly transformed into design parameters meanwhile. At the same time, morphological analysis is used to express the relationship between the function requirements and design parameters. Then, the patent analysis is used to select the best combination of design parameters as the product design scheme. Finally, the independent axiom in axiomatic design is used to evaluate the scheme.

\section{Design frameworks}

\subsection{Patent analysis (PA)}

The global patent data set is big by any measure, with millions of new patent documents and updates made public every week. According to World Intellectual Property Organization report, just in 2018, patents applicated in US is more than 30,000 . For technology and intellectual property(IP) professionals, weeding through this data to determine new business opportunities and/or competitive risks is a crucial task. Patent data is well suited for big data tools and techniques because of the volume, variety and velocity of changes(Gutierrez, 2016). In fact, many of today's patent analytics solutions are leading the way in the use of big data to drive market analysis, product development.

Patents analysis in this paper include three steps. The first and most important step is patent retrieval by classification numbers and keywords. Classification number is structured data which included International Patent Number(IPC), Combined Patent Number(CPC), US Patent Number(UPC) and F-term. Keywords are mainly about text in title, abstract, claims and background of patents. The second step is data analysis, which mainly analyses the structure or principle and its functions of each patent. This progress is carried out by expert and text mining technology(Noh and Jo et al., 2015). The third step is data statistics, which is to calculate the frequency of patents related to the structure, principle and function in the second step.

\subsection{Morphological analysis (MA)}

The general morphological analysis which was first developed by Zwicky to retrieve all possible solution for a multi-dimensional, non-quantifiable problem where causal modelling and simulation do not function well(Ritchey, 2011), where in every dimension one solution is possible, is one of the best technic of retrieving candidate solution for a multi-dimensional problem(Moubachir and Bouami, 2015). A general form of MA is using matrix as a method to structuring and investing the total set of relationships contained in problem complex and has been applied to diverse fields(Silin and Patzek, 2006).

However, MA depends on expert's intuition and does not have any systematic method for dividing problem and identifying dimensions (Yoon and Park et al., 2014), and cannot used in product development alone. In this research, $\mathrm{AD}$ and $\mathrm{PA}$ are incorporated to remedy the limitation of MA and the integrated model is proposed in section 2.3.

\subsection{The proposed model}

This section discusses the proposed methodology for supporting Zigzag mapping progress of $\mathrm{AD}$ and selecting the best solution by patent analysis and morphologic analysis. Since FRs and DPs are two most important components of axiomatic design, and designers should always make an effect to mapping them. This section details a model used to map between FRs and DPs in AD. The aim of the model is to remedy the shortage of AD by PA and MA. And the model is illustrated in Fig 2. With the application of PA, information from patents can expand the knowledge for design, and DPs can be easily formed. More and this, PA can help designer to choose suitable DPs by patent data. And MA are used to combined design options into a set to aid designers come up with ideas. In order to describe all aspects of the research, a comprehensive design framework of the proposed model integrated AD, PA and MA is constructed, and illustrated in Fig 2.

According to the framework, the proposed design procedure is explained as in follow steps:

\section{Step 1: transform CRs into FRs}

Product designer firstly collects the requirements of customers by market research, then omits unrelated requirements and personal preference for identify the essential design problems, lastly the design problems are abstracted into FRs.

Step 2: make search queries of patents

Based on the FRs in step 1, firstly consider whether there are relevant patent classification numbers which 
include IPC, CPC, UPC and F-term, and IPC and CPC are used more frequently. In addition, keywords-based retrieval will be considered. Both patent classification number and keywords used for patent queries, there should be related to the FRs. The search result will be downloaded in local PC and imported into database.

\section{Step 3: decompose FRs into sub FRs}

The purpose of decomposing functions into sub-FRs is to detail the design objectives so as to improve the design efficiency. By using the zigzag mapping method in Axiomatic Design Theory and combining the results of the second step of PA, the total functional requirements can be decomposed into sub-FRs. Then sub-FRs are connected with flows to make function structure diagram, and the flows are materials, energy or information that is used by product.

\section{Step 4: search design parameters}

According to each sub-function-requirement, if there are related patents found in database, design parameters will be extract from patents by text mining. If there are not patents, the patents quires of patents will be remake, or the DPs will be given by experts.

Step 5: construct multi-dimension matrix of subFRs and DPs

A multi-dimension matrix is constructed to record the result of step 4. FRs are listed on the vertical axis of the matrix and DPs match the sub FRs are listed on the horizontal direction shown in Table 1.

Table 1. Morphological matrix

\begin{tabular}{clll}
\hline Dimension & & \multicolumn{2}{c}{ DPs } \\
\hline Sub-FRs 1 & DPs 11 & DPs 12 & $\ldots$ \\
Sub-FRs 2 & DPs 21 & DPs 22 & $\ldots$ \\
$\ldots$ & $\ldots$ & $\ldots$ & $\ldots$ \\
Sub-FRs n & DPs n1 & DPs n2 & $\ldots$ \\
\hline
\end{tabular}

\section{Step 6: propose and emulate design scheme}

Frequency of patents which include DPs in matrix will be measured and taken as the weight of DPs. According to each sub-FRs, the DPs with higher weight will be chosen and combined with other DPs to make new design schemes. Lastly, independent axiom of AD will be used to evaluate the performance of each design schemes and the best design will be obtained with the help of expert.

\section{Case study}

The following case study demonstrated one practical application of the design framework outlined in the previous section.

\subsection{Background}

With the improvement of life level, more and more consumers like to add essential oils, herbs, bath fluids and other materials in the bath, in order to enjoy the functions of bath and spa at the same time. Therefore, the development of spa functions of flower sprinkler has become a task for many bathroom enterprises, in the development of products at the same time, also applied for a large number of patents. Although some enterprises have developed the products and applicated patents to protect the ideas, the products can't adjust the flow of mixture of water and other materials. In this situation, entrusted by a local enterprise, we are assisting in the design of an essential oil spray that can surpass existing products and avoid infringement of the patents.

\subsection{Function analysis and patent queries}

Firstly, CRs are translated into FRs which include water spraying function and spraying agent function. According to those functions, the classification number is retrieved in advance, and the Boolean operation is combined to obtain the retrieval formula show in Table 2. We obtained spa shower patented granted from 1985 to 2018 in the USPTO database through Pantsnap, a famous commercial patent database service. We located the initial set of 162 patents from database. Then, after excluding irrelevant patents from the initial patents set, we obtain 127 valid patents related to spa shower.

By analysing the patents, FRs in table 2 are decomposed into small and easily solved sub-FRs, then FRs are used to make function structure diagram shown in Fig 3. The sub-FRs related to mixture are FR21 (fill tank), FR22(fix tank), FR23(flow out), FR24(adjust flow) and FR13(mix).

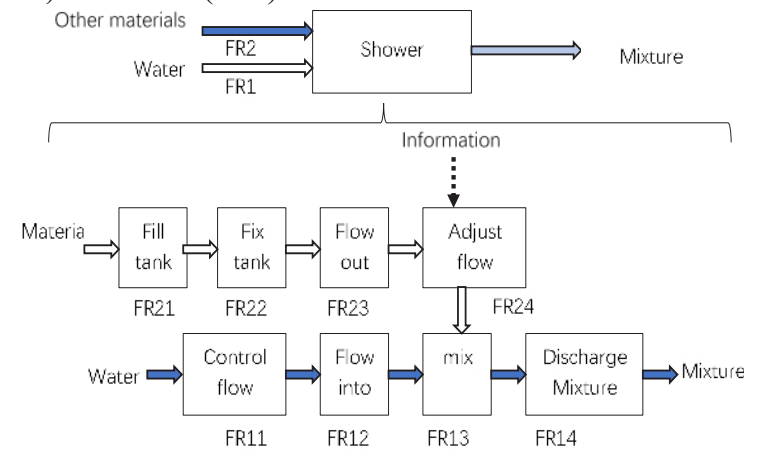

Fig. 3. Spa function structure diagram

\subsection{Design parameter analysis}

Based on text mining technology, all patents are analyzed to find out the design parameters matching subFRs, and the frequency of patents containing design parameters is counted. All the information is recorded in morphological matrix shown in Table 3. According to the frequency, we choose two DPs set as candidate of design solution. The first set include fluid, boxconnected way, embed way, knob, head and straight drawn way. The second set include fluid, box-packed way, embed way, knob, head and straight drawn way. Considered the advice of expert, we choose the second set as design object in next step. And the structure flow diagram shown in Fig 4. 


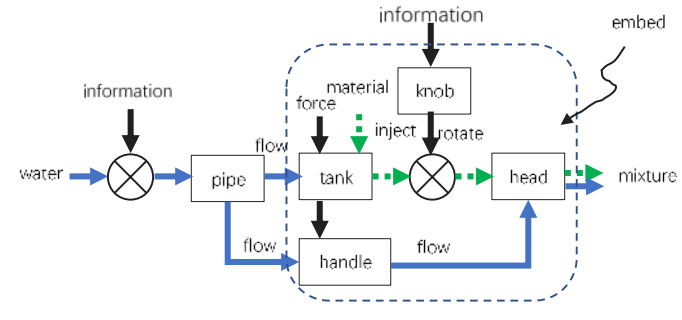

Fig. 4. Structure flow diagram

\section{4 spa shower development base on AD}

By using zigzag method of AD, sub-FRs in second set of spa shower are decomposed into 19 FRs shown in Fig 4. According the FRs shown in Fig 5, DPs are mapped and shown in Fig 6.

Design solution is shown in Fig 7. The shower can inject mixture of water and other materials, and the mixture flow can be adjusted by knob. Moreover, the shower using water draw material stored in tank which is embed in handle, so the materials can be fluid, solid, grain, powder and even mucus. If materials are using up, tank can be taken apart from shower easily by hand. Tank is embedded in handle for making streamlined shaper of shower.

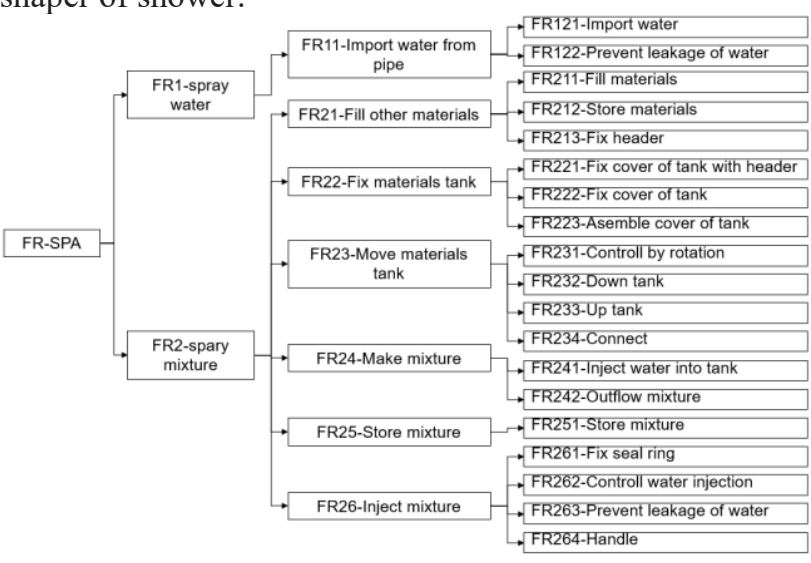

Fig. 5. Further of decomposition of sub FRs

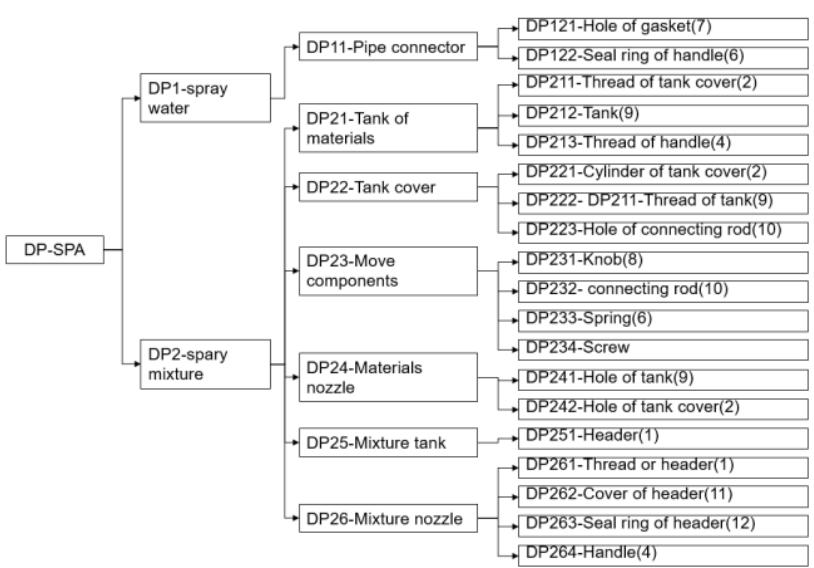

Fig. 6. Further of decomposition of sub DPs

Principle of mixture flow adjusted function is shown in Fig 8. When connecting rod is listed, and tank will be listed by spring too. Then the hole in tank will be taken apart from gasket and water can flow into tank to mixed with materials. If connecting rod is pulled, the hole in tank will be embed in gasket, then water can't flow into tank where shower still can inject water.

Lastly, dependent axion is used to evaluated the performance of the design solution. Design matrix is structured to shown the couple relationship of FRs and DPs shown in Table 4. Form the table, we can find this spa shower is an uncoupled design, and don't has couple problem.

\section{Conclusion}

In this study, the $\mathrm{AD}$ is integrated with patent analysis technology and morphological matrix to support new product development first. Patent analysis not only provides much more information than expertise for mapping progress between function requirements and design parameters, but also offer more objective evaluation criteria than experts for selection of design parameters by patent's frequency. Morphological matrix is used to collect design parameters and show the relationship between function requirements and design parameters. The effectiveness of the proposed progress model is demonstrated by an illustrative example. In the future, we will continue to focus on the improvement of efficiency of axiomatic design method.

\section{Acknowledgements}

This work is supported by the National Natural Science Foundation of China(No. 51875220) and Fujian Provincial Nature Science Foundation of China (NO. FJ2017J0102). 
Table 2. FRs and patent retrieval query for spa shower

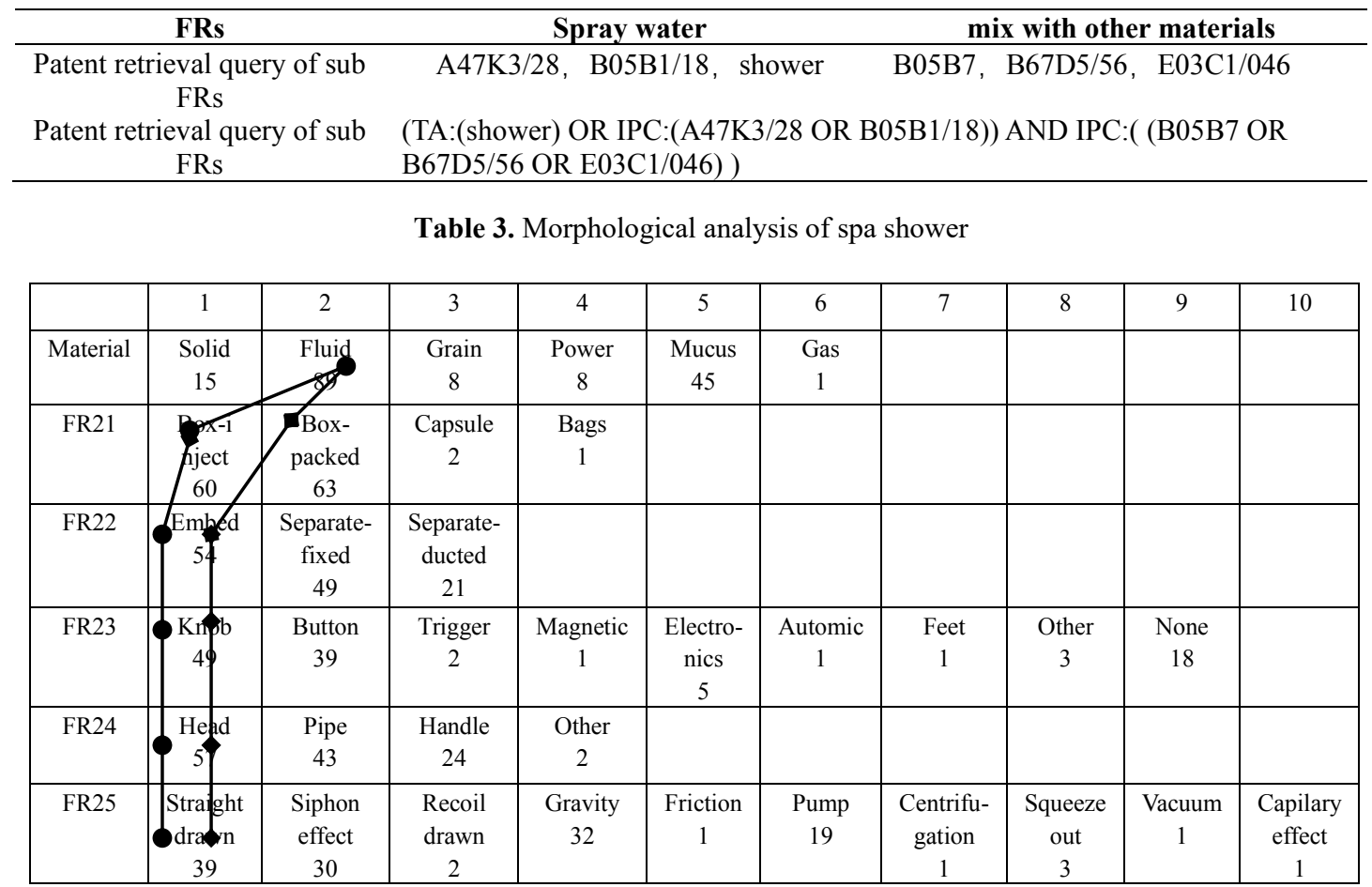

Table 4. Design Matrix of spa Shower

\begin{tabular}{|l|l|l|l|l|l|l|l|l|l|l|l|l|l|l|l|l|l|l|l|}
\hline & $\begin{array}{l}\text { DP1 } \\
21\end{array}$ & $\begin{array}{l}\text { DP1 } \\
22\end{array}$ & $\begin{array}{l}\text { DP2 } \\
11\end{array}$ & $\begin{array}{l}\text { DP2 } \\
12\end{array}$ & $\begin{array}{l}\text { DP2 } \\
13\end{array}$ & $\begin{array}{l}\text { DP2 } \\
21\end{array}$ & $\begin{array}{l}\text { DP2 } \\
22\end{array}$ & $\begin{array}{l}\text { DP2 } \\
23\end{array}$ & $\begin{array}{l}\text { DP2 } \\
31\end{array}$ & $\begin{array}{l}\text { DP2 } \\
32\end{array}$ & $\begin{array}{l}\text { DP2 } \\
33\end{array}$ & $\begin{array}{l}\text { DP2 } \\
34\end{array}$ & $\begin{array}{l}\text { DP2 } \\
41\end{array}$ & $\begin{array}{l}\text { DP2 } \\
42\end{array}$ & $\begin{array}{l}\text { DP2 } \\
51\end{array}$ & $\begin{array}{l}\text { DP2 } \\
61\end{array}$ & $\begin{array}{l}\text { DP2 } \\
62\end{array}$ & $\begin{array}{l}\text { DP2 } \\
63\end{array}$ & $\begin{array}{l}\text { DP2 } \\
64\end{array}$ \\
\hline FR251 & 1 & & & & & & & & & & & & & & & & & & \\
\hline FR261 & & 1 & & & & & & & & & & & & & & & & & \\
\hline FR211 & & & 1 & & & & & & & & & & & & & & & & \\
\hline FR242 & & & & 1 & & & & & & & & & & & & & & & \\
\hline FR221 & & & & & 1 & & & & & & & & & & & & & & \\
\hline FR122 & & & & & & 1 & & & & & & & & & & & & & \\
\hline FR264 & & & & & & & 1 & & & & & & & & & & & & \\
\hline FR213 & & & & & & & & 1 & & & & & & & & & & & \\
\hline FR212 & & & & & & & & & 1 & & & & & & & & & & \\
\hline FR222 & & & & & & & & & & 1 & & & & & & & & & \\
\hline FR241 & & & & & & & & & & & 1 & & & & & & & & \\
\hline FR233 & & & & & & & & & & & & 1 & & & & & & & \\
\hline FR121 & & & & & & & & & & & & & 1 & & & & & & \\
\hline FR223 & & & & & & & & & & & & & & 1 & & & & & \\
\hline FR231 & & & & & & & & & & & & & & & 1 & & & & \\
\hline FR234 & & & & & & & & & & & & & & & 1 & & & \\
\hline FR232 & & & & & & & & & & & & & & & & 1 & & \\
\hline FR262 & & & & & & & & & & & & & & & & & & 1 & \\
\hline FR263 & & & & & & & & & & & & & & & & & & & 1 \\
\hline
\end{tabular}




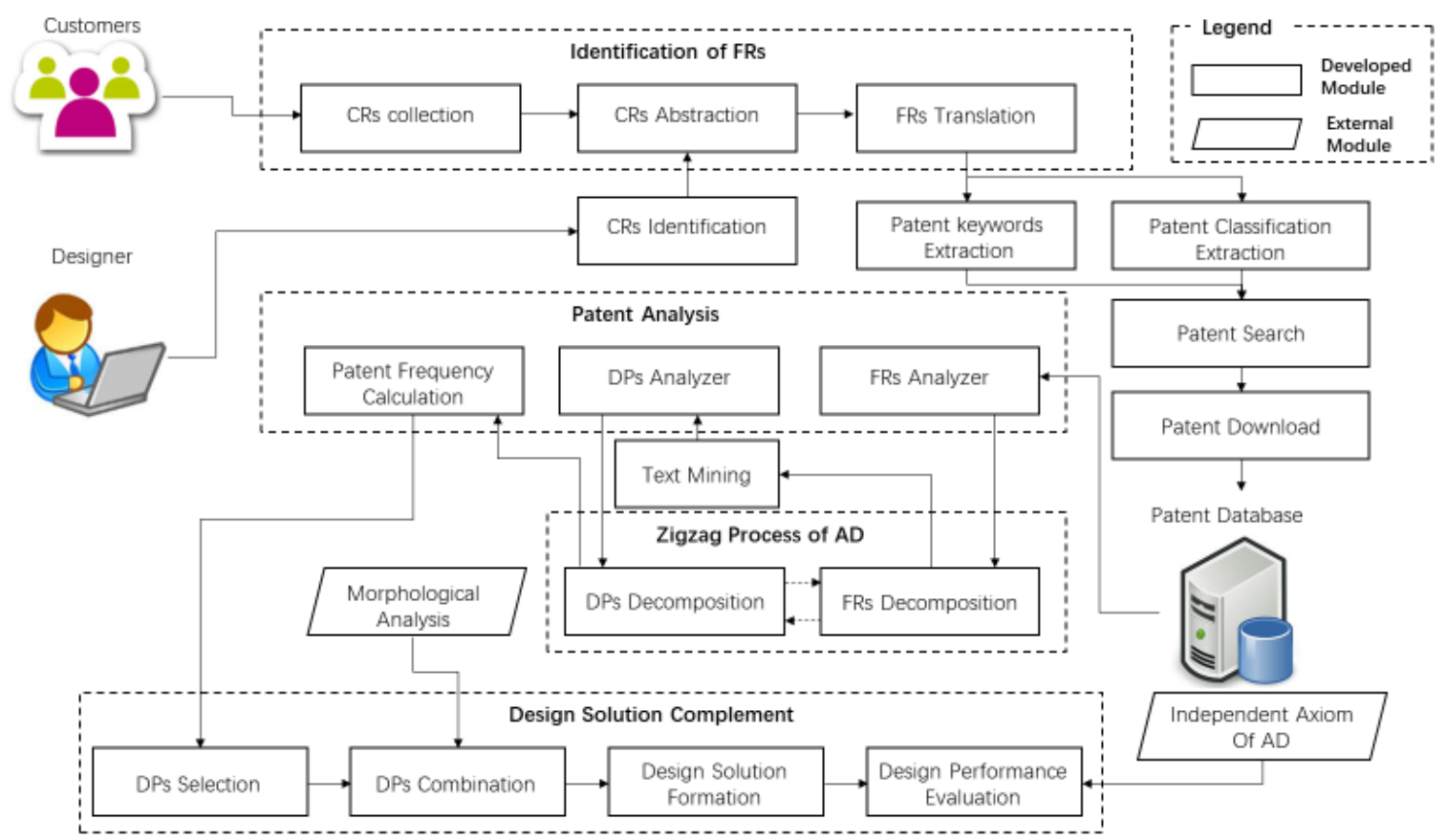

Fig. 2. Framework of the proposed model
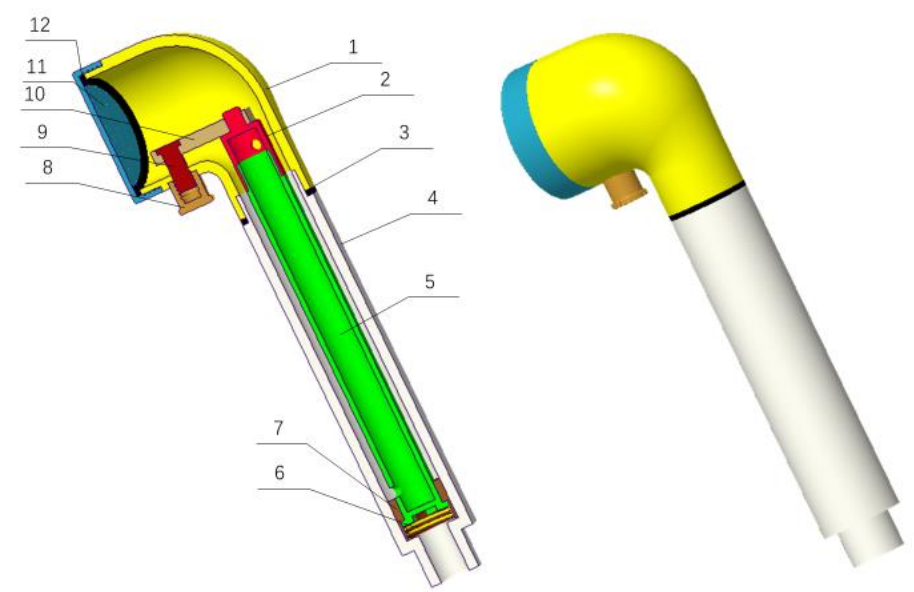

(a) Inner structure of spa shower

(b) External view of spa shower

1-header; 2-cover of tank; 3-seal ring of handle; 4-handle; 5-tank; 6-spring; 7-gasket; 8-knob; 9-screw; 10-connecting rod ; 11-cover of header; 12 -seal ring of header

Fig. 7. Spa Shower

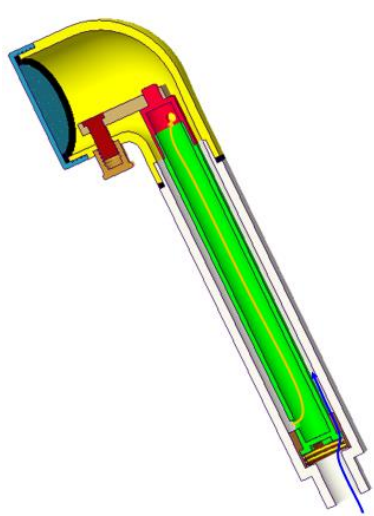

(a) Mixture injection principle

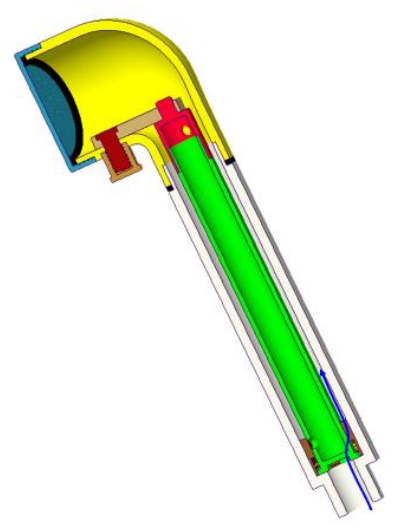

(b) Water injection principle

Fig. 8. Principle of spa Shower 


\section{References}

1. Suh (2001). Axiomatic Design: Advances and Applications, Oxford University Press.

2. Li, W. and Z. Song, et al. (2019). "Using Extenics to describe coupled solutions in Axiomatic design." Journal of Engineering Design 30 (1): 1-31.

3. Wang, H. and X. Zhang, et al. (2017). "A semantic model for axiomatic systems design." Proceedings of the Institution of Mechanical Engineers, Part C: Journal of Mechanical Engineering Science 232 (12): 2159-2184.

4. Kumar, P. and P. Tandon (2019). "A paradigm for customer-driven product design approach using extended axiomatic design." Journal of Intelligent Manufacturing 30 (2): 589-603.

5. He, Y. and C. Zhu, et al. (2017). "Big data oriented root cause identification approach based on Axiomatic domain mapping and weighted association rule mining for product infant failure." Computers \& Industrial Engineering 109: 253-265.

6. Yan, W. and C. Chen, et al. (2009). "A data-mining approach for product conceptualization in a webbased architecture." Computers in Industry 60 (1): 21-34.

7. Ireland, R. and A. Liu (2018). "Application of data analytics for product design: Sentiment analysis of online product reviews." CIRP Journal of Manufacturing Science and Technology 23: 128144.

8. Schaefer, D. (2014). Cloud-Based Design and Manufacturing (CBDM). DE, Springer Verlag.

9. Chang, S. W. C. and C. V. Trappey, et al. (2014). "Forecasting Dental Implant Technologies Using Patent Analysis." $2014 \quad$ PORTLAND INTERNATIONAL CONFERENCE ON MANAGEMENT OF ENGINEERING \& TECHNOLOGY (PICMET): 1483-1491.

10. Gutierrez, D. (2016). "Big Data Transforms Patent Analysis into Business Strategy." insideBIGDATA.

11. Noh, H. and Y. Jo, et al. (2015). "Keyword selection and processing strategy for applying text mining to patent analysis." Expert Systems with Applications 42 (9): 4348-4360.

12. Ritchey, T. (2011). General morphological analysis (GMA). Berlin, Springer.

13. Moubachir, Y. and D. Bouami (2015). "Storing and Retrieving Design Solution in the Physical Domain Based on DFX Tools and Morphological Analysis." Procedia CIRP 34: 64-68.

14. Silin, D. and T. Patzek (2006). "Pore space morphology analysis using maximal inscribed spheres." Physica A: Statistical Mechanics and its Applications 371 (2): 336-360.

15. Yoon, B. and I. Park, et al. (2014). "Exploring technological opportunities by linking technology and products: Application of morphology analysis and text mining." Technological Forecasting and Social Change 86: 287-303. 